\title{
Elaboration of double emulsion-based polymeric capsules for fragrance
}

\author{
Margot Stasse ${ }^{1,2}$, Tiphaine Ribaut ${ }^{3}$, Valérie Héroguez ${ }^{2 *}$, Véronique Schmitt ${ }^{1 *}$ \\ ${ }^{1}$ Centre de Recherche Paul Pascal UMR 5031 CNRS University of Bordeaux France \\ ${ }^{2}$ Laboratoire de Chimie des Polymères Organiques UMR 5629 CNRS University of \\ Bordeaux France \\ ${ }^{3}$ Takasago Europe Perfumery Laboratory 12 rue Torricelli 75017 Paris France \\ * corresponding authors : valerie.heroguez@enscbp.fr, veronique.schmitt@.crpp.cnrs.fr
}

\begin{abstract}
:
We aim at encapsulating fragrances made of a variety of lipophilic species to slow down their diffusion. Our strategy is to develop capsules by polymerizing the water intermediate phase of an oil-in-water-in-oil double emulsion. In other terms, our system consists in a direct emulsion of fragrance $(\mathrm{O} 1)$ in a water phase $(\mathrm{W})$ containing monomer, initiator and cross-linker. To obtain the double emulsion, this direct emulsion, stabilized by a hydrophilic surfactant, is itself dispersed in an external lipophilic solvent used in perfumery $(\mathrm{O} 2)$ and stabilized by a lipophilic surfactant. Polymerization of the intermediate water phase aims at obtaining a three dimensional network. Differently from nowadays-proposed capsules, this strategy allows polymerization only taking place in the water phase rather in the phase containing the fragrance. Moreover, the obtained 3D network is supposed to play the role of an effective barrier limiting the diffusion of the inner lipophilic species towards either the external solvent or air.

Such an approach implies the combination of a formulation step to elaborate the double emulsion using two antagonistic surfactants, a hydrophilic one and a lipophilic one, and of the polymerization of the intermediate phase. Insertion of the polymerizable species in the double emulsion shall not destabilize it. Some monomers exhibiting interfacial affinity and interfering with the formulation of the double emulsion have to be avoided. By varying the nature of the monomers and the cross-linker to monomer ratio, capsules with high encapsulation efficiencies and with various mechanical properties have been obtained.
\end{abstract}

Keywords: fragrance, capsules, encapsulation, double emulsions, free radical polymerization, monomers, cross-linkers, release, diffusion, network 


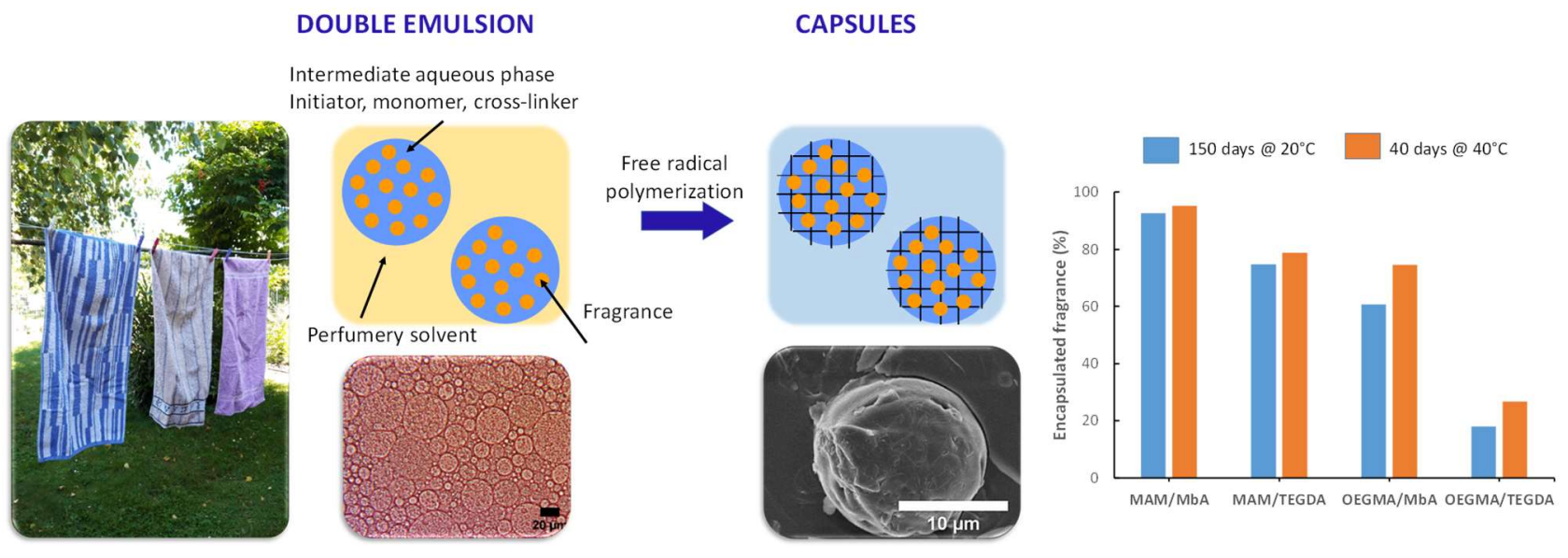

Polymerization of the intermediate aqueous phase of a fragrance-in-aqueous-phase-inperfumery solvent allows separating the polymerization locus from the fragrance compartment. This leads to capsules dispersible in water whose release properties can be tuned by the nature and concentration of monomer and cross-linker 


\section{Introduction}

Encapsulation of fragrances concern various applications such as detergency, cosmetics, pharmaceutics, foods, textiles, agriculture, building and so on [1-6]. Once deposited on a substrate, they act as a fragrance reservoir that can deliver their content. In the present work, the targeted application is encapsulation for softeners. For such an application, the specifications are the following: $i$ ) the same formulation should be valid for various fragrances, ii) the capsules should resist $\mathrm{pH}$ variations, iii) they should be $15 \mu \mathrm{m}$-sized to deposit on the textile, iv) they should be dispersible in water and $v$ ) fragrance is expected to be released during drying. Of course, all the compounds should be compatible with the application meaning that they should be non-toxic, non-irritant, non-mutagenic...

Today, capsules containing fragrance are already widely spread in detergents, cleaning and softeners [7-12]. Such capsules are usually synthesized by a suspension polymerization process $[7-10,12,13]$ meaning that the fragrance, the monomer and the initiator are miscible, and this blend is dispersed in water. Polymerization occurs during stirring. The obtained capsules are core-shell capsules made of a fragrance core and a polymer shell. They also often contain lattices in the fragrance. So the two main drawbacks are firstly the fact that, due to the miscibility with the fragrance, polymerization occurs in the fragrance phase while fragrance contains usually reactive molecules and secondly the presence of latex (Figure 1).

In the present work, we propose an alternate strategy based on a double $\mathrm{O} / \mathrm{W} / \mathrm{O}$ emulsion that is to say a fragrance-in-water-in-solvent double emulsions (Figure 1). This means that the aqueous globules contain fragrance droplets. The idea is to separate the place where the polymerization takes place from the fragrance phase. Therefore, double emulsions exhibit the advantage of being composed of two kinds of compartments with different affinities: more lipophilic compartments containing the fragrance and aqueous compartments where we aim at inducing polymerization. Once obtained these capsules likely need to be transferred into an aqueous phase. 


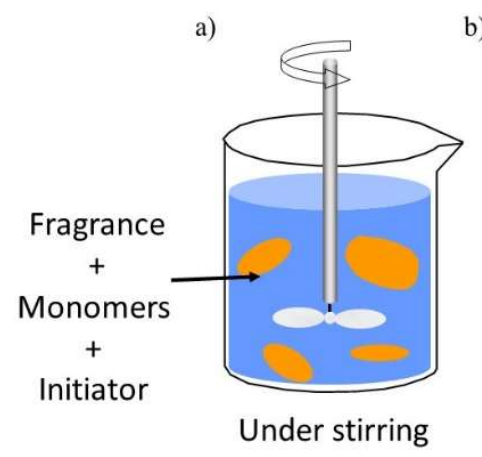

b)

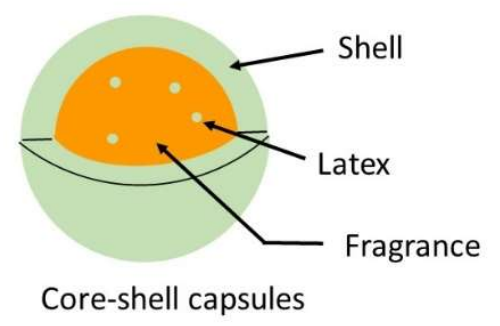

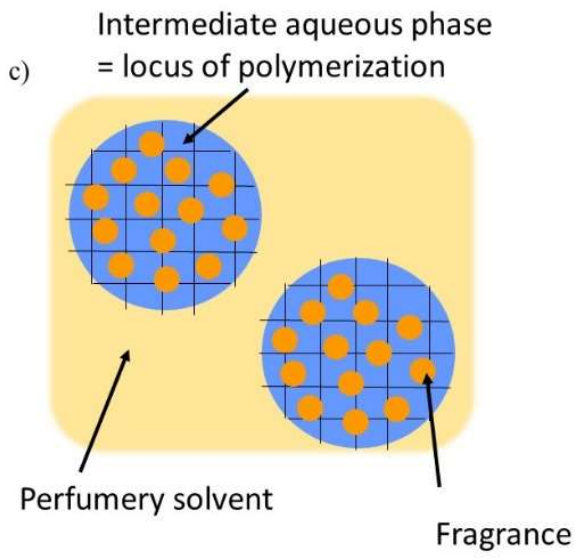

Figure 1: Schematic representation of a) and b) the nowadays most widely spread strategy to elaborate fragrance core-shell capsules and c) the alternate proposed strategy based on the polymerization of double emulsions for producing matrix capsules.

In the following, we will describe the progressive steps required to elaborate such capsules.

\section{Materials}

\subsection{Fragrances}

Two model fragrances noted A and B composed of 10 and 13 molecules respectively have been assembled by Takasago Europe Perfumery Laboratory to mimick real sytems. Their exact composition is given in supporting information S1. They include the classical class of molecules composing fragrances that is to say molecules with alkene, aldehyde, hydroxyl, ketone and ester moieties.

As a test to check the formulation resistance, we also used a third fragrance noted C. Its exact composition is also reported in $\mathrm{S} 1$.

\subsection{Hydrophilic Surfactants}

The selected hydrophilic non-ionic surfactants are: Tergitol 15-S-12 (molar mass $=760$ g.mol ${ }^{-1}$ and HLB = 14.5), Tween $60\left(\right.$ molar mass around 1310 g. $\left.\mathrm{mol}^{-1}, \mathrm{HLB}=14.9\right)$, Tween 20 (molar mass around 1220 g.mol ${ }^{-1}, \mathrm{HLB}=16$ ) all purchased from Sigma-Aldrich.

\subsection{Lipophilic Surfactant}

The selected lipophilic surfactants (LS) is polyglycerol polyricinoleate (PGPR) with a molar mass of 3000 g.mol-1 (HLB = 1.5) graciously provided by Paalsgard. 


\subsection{Perfumery solvents}

The solvents are water possibly containing $\mathrm{NaCl}$ and isopropyl myristate (noted IPM in the following, density equal to $0.850 \mathrm{~g} \cdot \mathrm{mL}^{-1}$ ) chosen because it is widely used in perfumery and graciously provided by Takasago Europe Perfumery Laboratory.

\subsection{Monomers}

Four monofunctionnal monomers have been selected for their hydrophilicity and the agreement to use them in cosmetics: Hydroxyethyl-methacrylate (98\%) noted HEMA, Oligo(ethyleneglycol) methacrylate noted OEGMA with $\mathrm{M}_{\mathrm{n}}=360$ g. $\mathrm{mol}^{-1}$ (containing 500-800 ppm of monomethyl ether hydroquinone), Methacrylamide noted MAM (98\%), and Methyl vinyl ketone noted MKV.

\subsection{Cross-linkers}

Two different cross-linkers, namely difunctional monomers, have been considered: Tetra(ethyleneglycol) diacrylate noted TEGDA and N,N-Methylene-bis-Acrylamide noted $\mathrm{MbA}$. The 4 units of ethylene glycol for TEGDA between the two acrylate functions is expected to confer flexibility to the tridimensional sought after network whereas the MbA can add physical cross-linking due to the possible hydrogen bonding [14].

\section{Methods}

\section{1) Optical and electronic microscopy observations}

Emulsions and capsules were observed with a Leica Leitz inverted optical microscope and the images were recorded with an IDS video camera and the Scion image program. The globule or the capsule size was estimated through a statistic analysis over about 50 particles.

For the capsules, scanning electron microscopy (SEM) pictures were taken with a Hitachi TM-1000 apparatus. To avoid deformation of the capsules possibly provoked by vacuum, Cryo-SEM was performed with a JEOL 6700FEG electron microscope equipped with liquid nitrogen cooled sample preparation and transfer units. A small sample of the dispersion of capsules was first deposited on the aluminum specimen holder. The sample was then frozen in the slushing station with boiling liquid nitrogen and transferred under vacuum from the slushing station to the preparation chamber. The latter was held at $\mathrm{T}=-150^{\circ} \mathrm{C}$ and $\mathrm{P}=10^{-5} \mathrm{~Pa}$ and was equipped with a blade used to fracture the sample. Once fractured, the sample was 
coated by a layer of Au-Pd and then inserted into the observation chamber equipped with a SEM stage cold module held at $-150^{\circ} \mathrm{C}$.

\section{2) Static light scattering}

The emulsions size distributions were measured using static light scattering by means of a Malvern Mastersizer MS2000 granulometer. The software gives access to the volumeaveraged drops size $\mathrm{D}[4,3]$ and to the polydispersity index called uniformity and noted $\mathrm{U}$ defined as the volume-average difference between the diameter and the median diameter normalized by the median diameter. Let us remind that the median diameter corresponds to the midpoint of the size distribution meaning the diameter for which half of the dispersed phase is distributed in drops smaller than the median diameter and the other half is distributed in drops larger than the median diameter.

\section{3) Gas chromatography}

Gas chromatography (GC) has been used to analyze and quantify the amount of fragrance inside or outside the emulsion and capsules. The gas chromatograph (Agilent GC 7890B) is equipped with flame ionization detector (FID) coupled with Mass spectrometer 5977A (MS), a split/splitless injector (1:40 split ratio) and a data system (Enhanced ChemStation MSD Chemstation). The detector and injector temperatures were set at $250^{\circ} \mathrm{C}$. A $30 \mathrm{~m} \times 250 \mu \mathrm{m}$ internal diameter capillary column coated with 5\%-phenyl poly(methylsiloxane) (0.250 $\mu \mathrm{m}$ film thickness) (HP-5) was used. The oven temperature was programmed from 50 ${ }^{\circ} \mathrm{C}(2 \mathrm{~min})$ to $280{ }^{\circ} \mathrm{C}(5 \mathrm{~min})$ at $10^{\circ} \mathrm{C} / \mathrm{min}$. Two microliters of the sample were injected. Fragrance molecules are identified with MS and quantified according to an external calibration with different solutions of fragrance in ethanol on FID.

\section{4) DSC}

The polymeric capsules were analyzed by Scanning Differential Calorimetric (DSC) with a DSC Q100 RCS apparatus. The protocol was the following: first an increase of temperature from $-80^{\circ} \mathrm{C}$ to $120^{\circ} \mathrm{C}$ with a speed at $10^{\circ} \mathrm{C} / \mathrm{mn}$ was applied followed by a decrease down to $-80^{\circ} \mathrm{C}$. The second cycle started at $-80^{\circ} \mathrm{C}$ up to $230^{\circ} \mathrm{C}$ with a speed of $10^{\circ} \mathrm{C} / \mathrm{mn}$ followed by a last decrease down to $-50^{\circ} \mathrm{C}$ at the same speed.

\section{5) Mechanical compression}


The resistance of capsules against pressure was estimated using a homemade device. It is composed of three parts. The first part is an empty cup where the capsules are deposited. The second is a hollow piston equipped with a circle-shaped stainless-steel filter of $5 \mu \mathrm{m}$. The filter only allows the transfer of IPM containing the non-encapsulated fragrance while the capsules are maintained at the bottom below the filter. The piston is hollow to allow the external phase recovery under pressure. The third part is the load of different weights to access different pressures: 100 and $150 \mathrm{~g}$ corresponding to $780 \mathrm{~Pa}$ and to $1170 \mathrm{~Pa}$ respectively.

The protocol for the measurement is the following: the capsules are put in the cup and covered by IPM $(1: 1)$. The piston is placed on the sample and the weight is deposited on it. The pressure is applied for at least one hour to reach a stationary state. Then the IPM with the possibly released fragrance is recovered thanks to the hollow piston while maintaining the pressure. This recovered phase is then analyzed by GC to quantify the possibly leaked fragrance in IPM.

\section{Results and discussion}

\subsection{Elaboration of emulsions}

Firstly, we need to formulate emulsions since we aim at polymerizing the intermediate aqueous phase of an established double emulsion. This is very different from the suspension polymerization where polymerization may be initiated under stirring, without need of stabilizing an emulsion that forms and destroys continuously. As double emulsions are complex morphologies composed of the two types of direct oil-in-water and reverse water-in-oil simple emulsions, these two types of emulsions need to be formulated before combining them.

\section{Simple direct emulsion}

Following the Brancroft rule [15], the direct fragrance-in-water emulsion requires the use of a more hydrophilic surfactant. A first selection led to the choice of three non-ionic surfactants namely Tween $20 \AA$, Tween $60 \AA$ and Tergitol $15-\mathrm{S}-12 \AA$ allowed in cosmetics. Since fragrance droplets have to be themselves dispersed in aqueous globules, the targeted droplet size is of the order of $1 \mu \mathrm{m}$.

The fragrance, corresponding to the dispersed phase of the direct emulsion, is progressively incorporated in the aqueous phase containing sodium chloride and the hydrophilic surfactant, up to a droplet mass fraction $\phi_{\mathrm{d}}=90 \%$. This crude emulsion is then sheared using a Couette cell applying a shear rate of $14280 \mathrm{~s}^{-1}$ during about $10 \mathrm{~s}$. We chose preparing highly concentrated emulsions in order to better reduce the average drop size and to narrow the drop size distribution 
$[16,17]$. Indeed, it has been demonstrated that shearing a concentrated emulsion is highly efficient. Also for dilute emulsions, the resulting drop size depends upon the interfacial tension: the lower the interfacial tension, the smaller the drop size [16]. More precisely, the size is proportional to the interfacial tension all the other parameters being kept identical, as a result of a constant critical capillary number defined as $\mathrm{Ca}_{\mathrm{cr}}=\frac{\sigma \mathrm{d}_{\mathrm{droplet}}}{2 \gamma}$ where $\sigma$ is the applied stress, $\mathrm{d}_{\text {droplet }}$ the obtained droplet size and $\gamma$ the interfacial tension. Obviously, this result remains valid in the present case of highly sheared concentrated emulsions (see Figure 2). As micrometric droplets are targeted to be incorporated in the double emulsion, we chose the Tergitol 15-S$12 ®$ surfactant for the following. The obtained emulsions exhibit a uniform drop size distribution as can be quickly visualized by the large field optical microscopy pictures and as can be quantified by the drops size distributions reported in Figure 2.
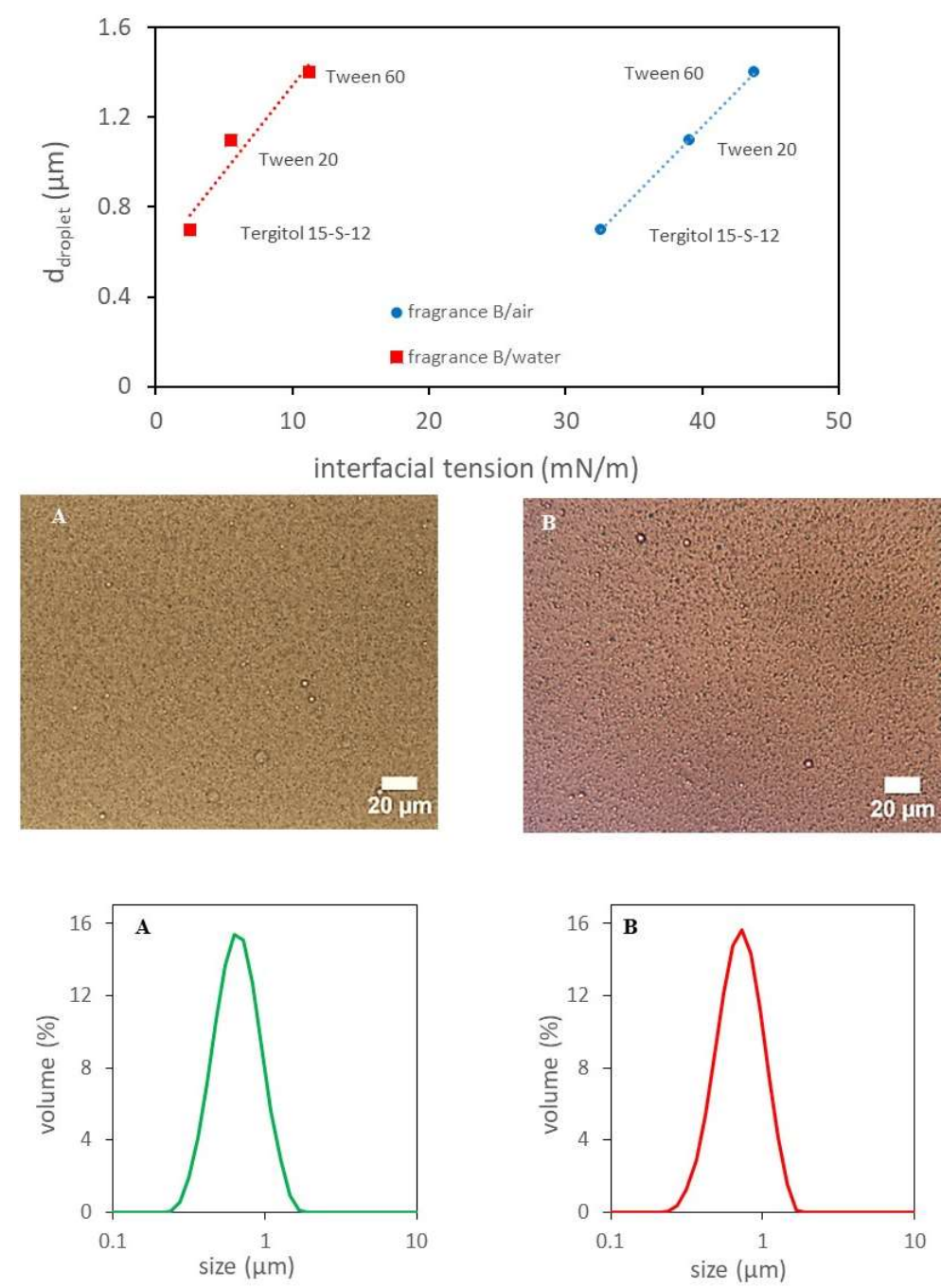

Figure 2: Result of the direct emulsion preparation, top) obtained droplet size as a function of the interfacial tension between fragrance B and brine or fragrance B and air in presence of the selected surfactant, middle) large view of the emulsions prepared with Tergitol 15-S-12 ${ }^{\circledR}$ and fragrance A (left) and B (right) and bottom corresponding droplet size distribution measured by 
static light scattering. In both cases, the volume-average droplet size is $0.7 \mu \mathrm{m}$ and the polydispersity index $\mathrm{U}$ is $28 \%$. Adapted from [18].

\section{Simple reverse emulsion}

For the reverse brine-in-IPM emulsion, the widely spread PGPR surfactant has been selected [19]. Brine $(0.1 \mathrm{M} \mathrm{NaCl})$ is preferred to pure water as it is known that adding salt in water is very efficient to prevent Ostwald ripening [20] in reverse emulsions. Ostwald ripening is one of the two mechanisms responsible for the drop size evolution with time in emulsions. It proceeds by molecular diffusion of the dispersed phase through the continuous phase due to its solubility. Indeed salted water is less soluble in oil than pure water. The emulsion is prepared by progressively adding brine into IPM containing $10 \mathrm{wt} \%$ of PGPR up to a mass fraction of globule $\phi_{\mathrm{G}}=75 \%$. This crude emulsion is then sheared at various shear rates. The targeted size should be of the order of $10-15 \mu \mathrm{m}$ as this range of size is thought to be efficient for deposition on textiles [21] (Figure 3) 

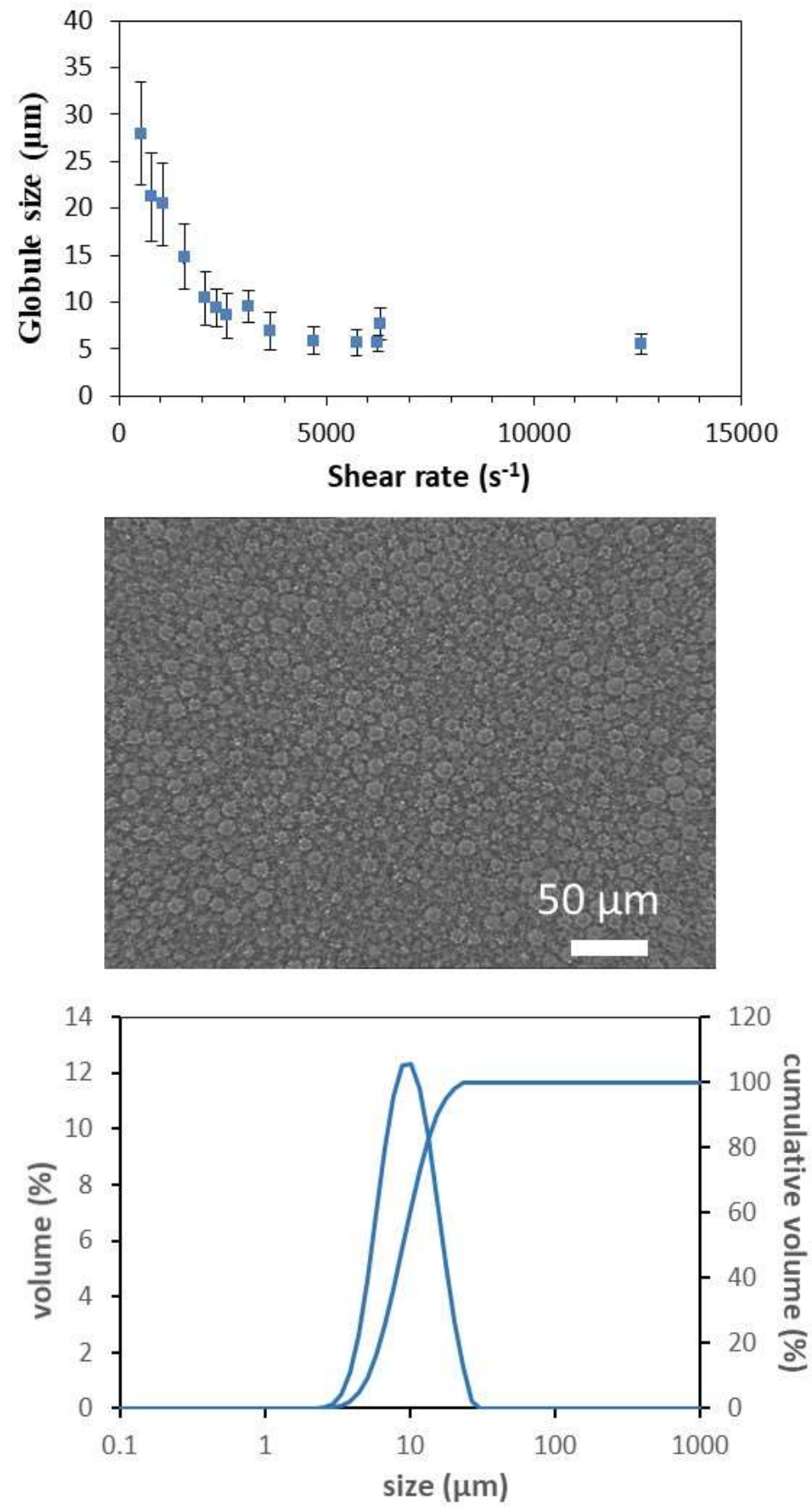

Figure 3: Result of the reverse emulsion preparation, top) obtained globule size as a function of the applied shear rate. The error bars represent the polydispersity index U, middle) Optical microscopy picture of the emulsion after shearing at $3150 \mathrm{~s}^{-1}$ and bottom corresponding globule size distribution measured by static light scattering. The volume-average globule size is $9.5 \mu \mathrm{m}$ and the polydispersity index $U$ is $35.5 \%$. Adapted from [18].

\section{Preparation of the $\mathrm{O} / \mathrm{W} / \mathrm{O}$ double emulsion}

The double fragrance A or B-in-brine-in isopropyl myristate emulsions are prepared by a two-step procedure. First, the direct fragrance in brine emulsion is prepared as described previously in the Simple direct emulsion section. This concentrated emulsion is then diluted 
with an aqueous solution containing or not additional Tergitol $15-\mathrm{S}-12 \AA$. This dilution allows fixing both the mass fraction of droplets $\phi_{\text {droplet }}$ as well as the composition of the intermediate aqueous phase (salt and surfactant concentrations). Secondly, this diluted direct emulsion is progressively incorporated in IPM containing $10 \mathrm{wt} \%$ of PGPR. The emulsions are then observed by optical microscopy to determine whether the emulsion is indeed double or if the aimed morphology is not obtained. All the results may be reported on ( $\phi_{\text {droplet }}$ - Tergitol 15-S12®) diagrams (Figure 4). The green squares correspond to formulations where double emulsion morphology is obtained. By comparing the diagrams from both fragrances, it can be noticed that the domains are the same for both fragrances showing the solidity of the formulation toward a fragrance variation. In order to even better test this resistance we prepared a double emulsion with a formulation in the green domain with the third fragrance C. Again a double emulsion morphology is observed.
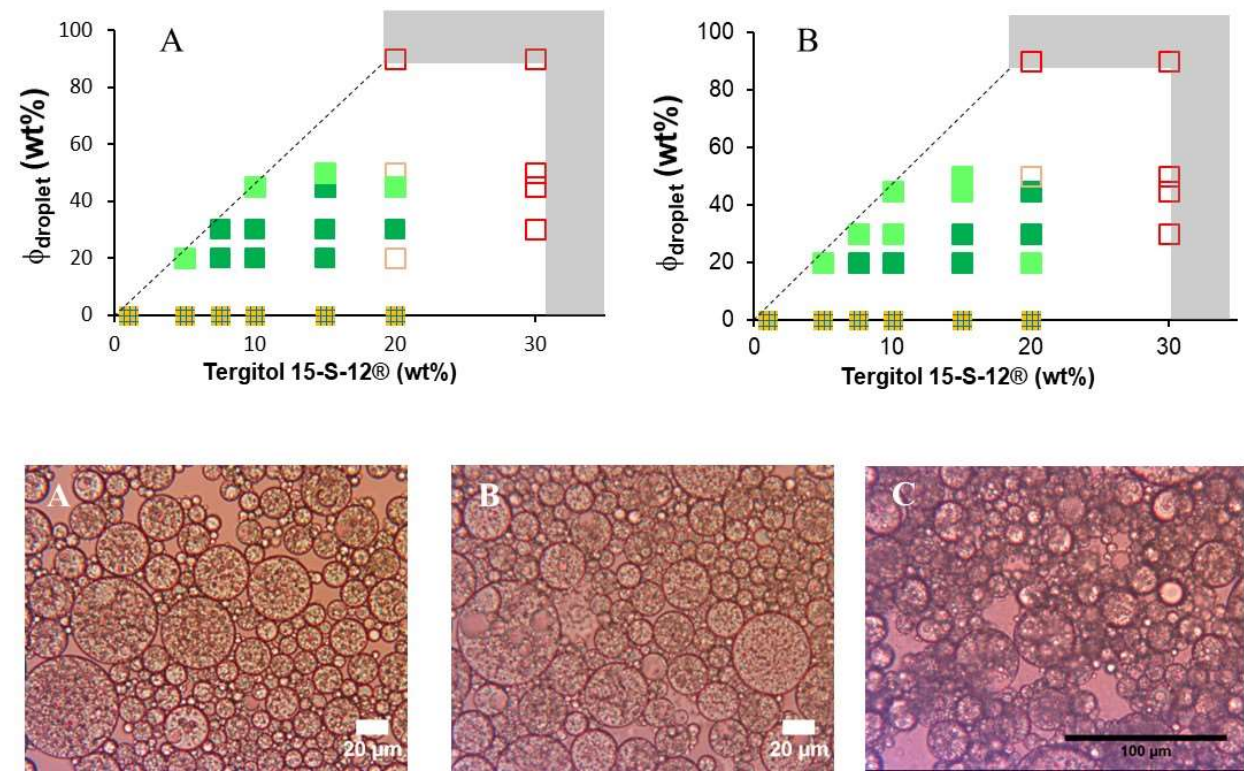

Figure 4: Feasibility of doubles emulsions: effect of the droplet fraction and the amount of hydrophilic surfactant keeping the fraction of PGPR $(10 \mathrm{wt} \%)$ and the globule fraction $\left(\phi_{\mathrm{G}}=75\right.$ wt $\%$ ) constant. Top) ( $\phi_{\text {droplet }}$ - Tergitol 15-S-12®) diagrams for fragrance A (left) and B (right). Caption: $(\square)$ very few multiple droplets $(\square)$ few multiple droplets in coexistence with simple ones $(\square$ ) only multiple droplets $(\square$ ) Multiple droplets with few simple ones $($ \#) multiple droplets (without fragrance) - _ - Limit of dilution of the direct emulsion. In grey, inaccessible area by the proposed two-steps process including a dilution of the direct emulsion.

Bottom) Double emulsions with $\left(\phi_{\text {droplet }}=30 \mathrm{wt} \%\right.$ and $15 \mathrm{wt} \%$ of Tergitol $15-\mathrm{S}-12 \AA$ for fragrance A, B and C from left to right. Adapted from [18].

This shows that the proposed composition of double emulsion may be used with all the various molecules composing these three fragrances, none of them is deleterious for preparation of the double emulsion. 
In the following, we will consider the emulsion composed of fragrance B, with $\phi_{\text {droplet }}=20 \mathrm{wt} \%$, $7.5 \mathrm{wt} \%$ of Tergitol $15-\mathrm{S}-12 \AA$ in brine, $\phi_{\mathrm{G}}=75 \mathrm{wt} \%, 10 \mathrm{wt} \%$ of PGPR in IPM. The details are given in Table 1.

Table 1: Composition of the selected double emulsion for the rest of the paper, the same composition is expressed differently. In the first row, the composition is given with respect to the considered phase. The last row gives the amounts for $100 \mathrm{~g}$ of total sample.

\begin{tabular}{|c|c|c|}
\hline Inner phase & Intermediate phase & Outer phase \\
\hline $100 \%$ of fragrance $\mathrm{A}, \mathrm{B}$ or $\mathrm{C}$ & $\begin{array}{l}7.5 \mathrm{wt} \% \text { of Tergitol } 15-\mathrm{S}-12 \AA \\
92.5 \mathrm{wt} \% \text { of brine }\end{array}$ & $\begin{array}{l}10 \% \text { of PGPR } \\
90 \% \text { of isopropyl myristate }\end{array}$ \\
\hline $\begin{array}{l}20 \% \text { with respect to the } \\
\text { intermediate phase }\end{array}$ & $\begin{array}{l}75 \mathrm{wt} \% \text { with respect to the total } \\
\text { sample }\end{array}$ & $\begin{array}{l}25 \mathrm{wt} \% \text { with respect to total } \\
\text { sample }\end{array}$ \\
\hline $15 \mathrm{~g}$ of fragrance $\mathrm{A}, \mathrm{B}$ or $\mathrm{C}$ & $\begin{array}{l}4.5 \mathrm{~g} \text { of Tergitol } 15-\mathrm{S}-12 \AA \\
55.5 \mathrm{~g} \text { of } \mathrm{NaCl} \text { brine }\end{array}$ & $\begin{array}{l}2.5 \mathrm{~g} \text { of PGPR } \\
22.5 \mathrm{~g} \text { of isopropyl myristate }\end{array}$ \\
\hline
\end{tabular}

\section{Encapsulation efficiency and composition of the encapsulated fragrance}

A major point is the determination of the encapsulation efficiency $\varepsilon$ defined as the proportion of fragrance that is inside the double emulsion.

$$
\varepsilon=\frac{\text { mass of encapsulated fragrance }}{\text { total mass of fragrance }}
$$

It is deduced from the determination of the non-encapsulated fragrance that is to say the fragrance that could be solubilized in the outer IPM. To assess the amount of fragrance in IPM, the emulsion is centrifuged at $1500 \mathrm{rpm}$ during $25 \mathrm{~min}$ to induce separation of the continuous phase from the emulsion without double emulsion rupture. The continuous phase is then recovered, filtrated and diluted in ethanol up to $5 \mathrm{wt} \%$. This phase is then analyzed by gas chromatography. The amount of fragrance in IPM is $1.05 \mathrm{wt} \%$ of initial amount leading to an encapsulation rate of $\varepsilon=98.5 \mathrm{wt} \%$. Usually, the encapsulated species is a unique molecule of interest but as fragrance B is a blend of 13 molecules, it is also of great interest to determine the composition of the encapsulated fragrance and to compare it with the initial fragrance. The results obtained by gas chromatography are reported on Figure 5. 


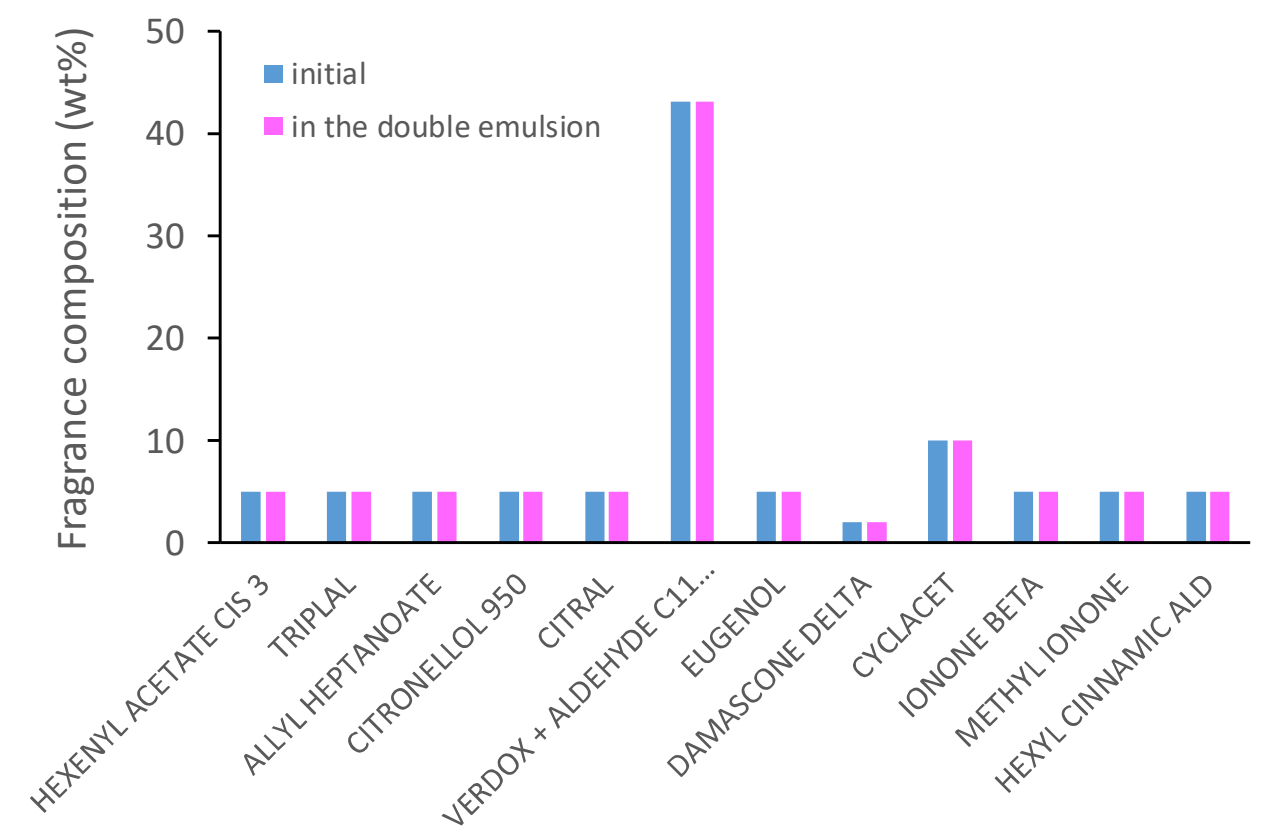

Figure 5: composition of bulk (initial) and encapsulated (in the double emulsion) fragrance B.

This means that the double emulsion allows a high encapsulation rate and does not alter the fragrance.

\subsection{Polymerization of the emulsions}

Again, we chose a progressive strategy to select the monomer for the aqueous phase polymerization beginning with the simple reverse emulsion. The composition is the same as previously that is to say $10 \%$ of PGPR in IPM for the continuous phase and $75 \mathrm{wt} \%$ of brine, the only difference is the use, in the aqueous phase, of KPS that will be used as initiator of the free radical polymerization.

Note that, at this point, neither the study of the kinetics of polymerization, nor the optimization of the conversion are sought after. However, even if not optimized, the conversion $\mathrm{C}$ is assessed by gravimetric measurements using:

$$
\mathrm{C}=\frac{\text { mass of optained polymer } \mathrm{x} \text { solid content by ATG }}{\text { proportion of monomer } \mathrm{x} \text { collected mass }}
$$

where the mass of obtained polymer is determined by weighting the purified and dried polymer, subtracting the non-volatile substances (surfactants and salt), the solid content by ATG takes 
into account the possibility of remaining water despite the lyophilization, the proportion of monomers represents the initial weight percentage of monomer initially present in the sample and the collected mass is the considered sample mass after polymerization.

\section{Polymerization of the reverse simple emulsion}

A first selection of non-CMR hydrophilic monomers leads to the choice of four monomers: 2-Hydroxyethyl methacrylate (HEMA), Methyl vinyl ketone (MVK), Methacrylamide (MAM) and Oligo(ethyleneglycol) methacrylate (OEGMA 360). In all cases, KPS has been chosen as initiator. Note that when KPS is used, the amount of $\mathrm{NaCl}$ is reduced as KPS releases ions. A first criterion to be tested is the possibility to incorporate the monomers in the reverse emulsion.

HEMA: The introduction of HEMA at $25 \mathrm{wt} \%$ and $50 \mathrm{wt} \%$ with respect to the aqueous phase leads to a strong decrease of the globule size to $7.1 \mu \mathrm{m}$ and $4.9 \mu \mathrm{m}$ respectively. This size decrease can be understood by a strong decrease of the interfacial tension between water and IPM (38.1 mN.m $\left.{ }^{-1}\right)$ and between water containing $25 \mathrm{wt} \%$ of HEMA and IPM (5.8 mN.m ${ }^{1}$ ) showing the strong affinity of HEMA towards the water/oil interface. As we are looking for polymerization to occur in the bulk water phase, HEMA is not a good candidate.

MVK: Addition of MVK in the aqueous phase does not modify the globule size of the reverse emulsion. However, it can be observed that the reverse emulsion sediment and coexist with a yellowish liquid at the top of the emulsion. This liquid is composed of IPM that is colorless and MVK that gives its yellow color. This means that MKV migrates into IPM and therefore is in fact too hydrophobic.

OEGMA 360: The introduction of $25 \mathrm{wt} \%$ of OEGMA in the aqueous phase does almost not alter the globule size $(8.4 \mu \mathrm{m})$. In addition, the size does not evolve over a period of 18 hours large enough to induce polymerization by heating the sample 24 hours (the time optimization was not the goal) at $70^{\circ} \mathrm{C}$. Polymerization is performed in a flask with a magnetic stirring and is quenched by exposure to air. The determined conversion is equal to $91 \%$. The obtained capsule size is very similar to the initial double emulsion globule size showing that no emulsion destabilization occurred during polymerization and no coagulum was formed.

Due to the very low solubility of polyOEGMA in usual solvents available for NMR or SEC, the polymer was analyzed by DSC. The DSC curve of the synthesized polymer exhibits a glass temperature $\left(\mathrm{Tg}=-53^{\circ} \mathrm{C}\right)$, a crystallization temperature $\left(\mathrm{Tc}=-8^{\circ} \mathrm{C}\right)$ and a melting point 
$\left(\mathrm{Tm}=7^{\circ} \mathrm{C}\right)$ characteristic of polyethylene glycol (PEG) chains. Comparison with the OEGMA macromonomer [22] shows a significant increase of glass temperature $\left(\mathrm{Tg}=-61^{\circ} \mathrm{C}\right)$ that can be understood as a decrease of the mobility of the OEGMA grafted chains by comparison with free chains, therefore attesting that the polymerization occurred.

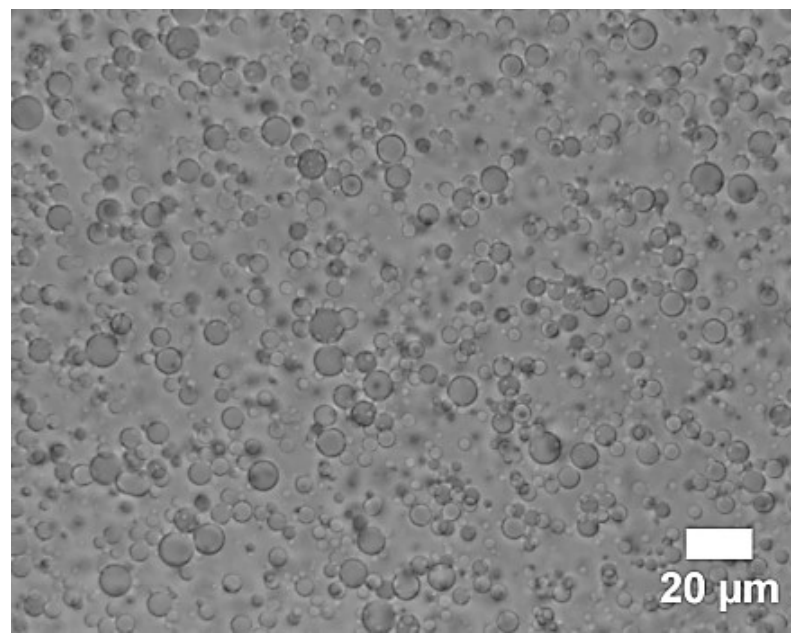

Figure 6: Optical microscopy of the polymerized simple reverse emulsion containing OEGMA. Adapted from [22].

MAM: The introduction of $15 \mathrm{wt} \%$ of MAM in the aqueous phase does not modify the globule size $(9.5 \mu \mathrm{m})$. In addition, the size does not evolve over a period of 18 hours large enough to induce polymerization by heating the sample at $70^{\circ} \mathrm{C}$. Polymerization is performed in a flask with a magnetic stirring for 24 hours (again the time optimization was not targeted) and is quenched by exposure to air. The determined conversion is equal to $64 \%$. The obtained capsule size is very similar to the initial double emulsion globule size showing that no emulsion destabilization occurred during polymerization and no coagulum was formed. Three heating/cooling cycles are performed (Figure 7). During the first cycle, a transition at $50-150^{\circ} \mathrm{C}$ is visible that disappears during the second and third cycles. This endothermic change is likely due to the evaporation of residual water. The endothermic change above $150^{\circ} \mathrm{C}$ can be attributed to physical cross-linking due to hydrogen bonding. This polyMAM chains physical crosslinking is validated by the non-solubility of the polymer except in strong acids known to be able to disrupt hydrogen bonds. 


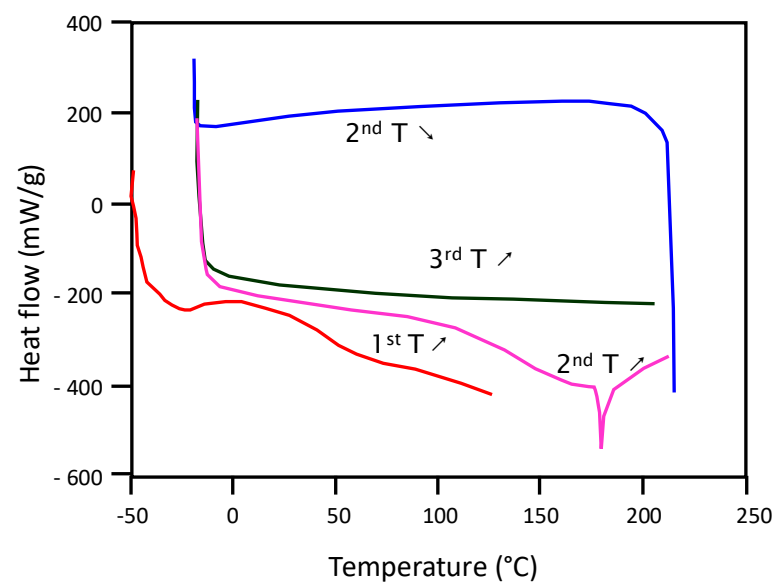

Figure 7: DSC analysis of the polymerized simple reverse emulsion containing MAM. Adapted from [22]

These polymerization tests in the reverse simple emulsion lead to the selection of the two MAM and OEGMA monomers for further use in the double emulsions.

\section{Polymerization of the double emulsions}

The following step consists in combining the previous results obtained in formulating double emulsions and polymerizing in simple reverse emulsion. In other words, double emulsions are prepared as previously described in presence either of $15 \mathrm{wt} \%$ of MAM or of 25 $w t \%$ of OEGMA. Polymerization is induced with the same protocol as applied to the reverse simple emulsion. Again, no coagulum is observed and capsules have sizes similar to the globule sizes of the double emulsions. More surprising, in opposition to the double emulsion globules, the obtained capsules are not dispersible in IPM but in water. This is likely linked to the nature of the obtained polymers, IPM is not a good solvent for the polyMAM or polyOEGMA. This means that no transfer of the capsules is required before use.

\section{Polymerization in the double emulsions in presence of cross-linkers.}

In order to elaborate a $3 \mathrm{D}$ network in the intermediate aqueous phase a cross-linker is added before polymerization. Two cross-linkers have been chosen again for their water affinity and non-toxicity: Methylene-bis-acrylamide noted $\mathrm{MbA}$ in the following and Tetra(ethylene glycol) diacrylate referred to as TEGDA in the following. A first formulation work consists in determining the maximum amount of cross-linker that can be incorporated in the double emulsion without altering the formula. The results are summarized in Figure 8a where the amount of cross-linker is given in mol\% with respect to total amount of monomer and cross- 
linker. The polymerization is initiated as at $70^{\circ} \mathrm{C}$ and lasts for 24 hours in a double envelope reactor with a mechanical stirrer operating at $100 \mathrm{rpm}$

The result of polymerization is summarized in Figure $8 \mathrm{~b}$ where CryoSEM picture are reported. The obtained conversions are also noted.

a)

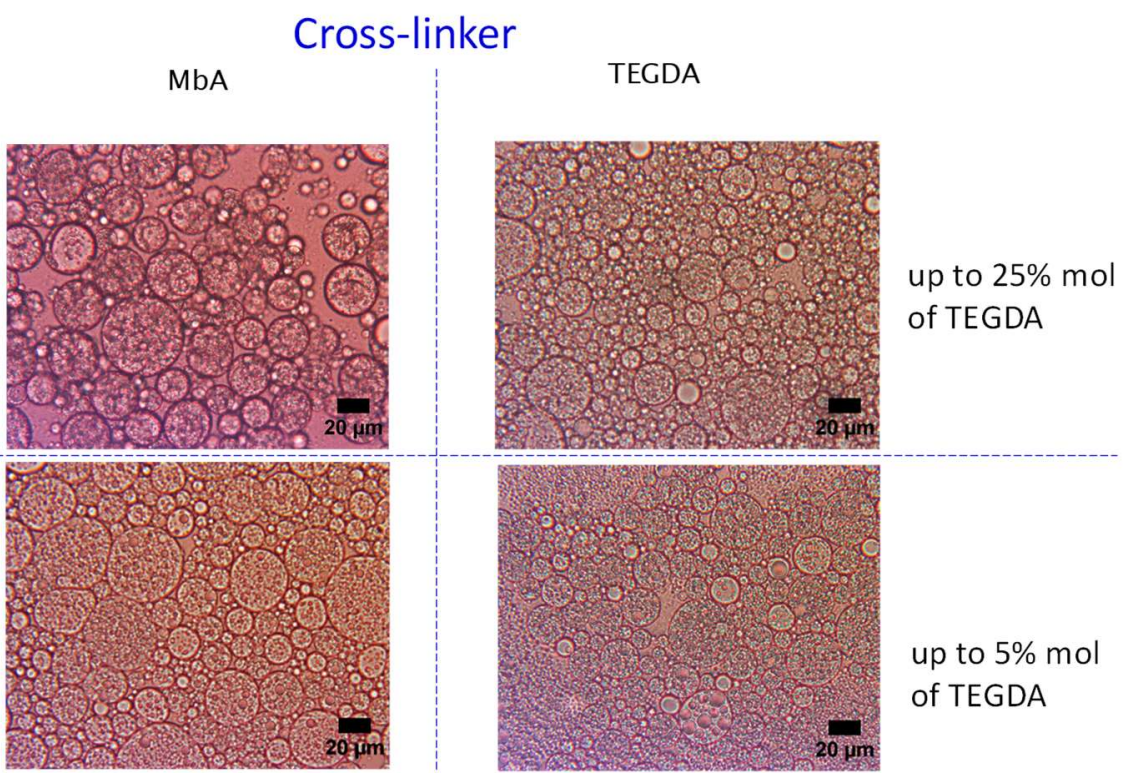

b)

\section{Cross-linker}
$\mathrm{MbA}$
TEGDA

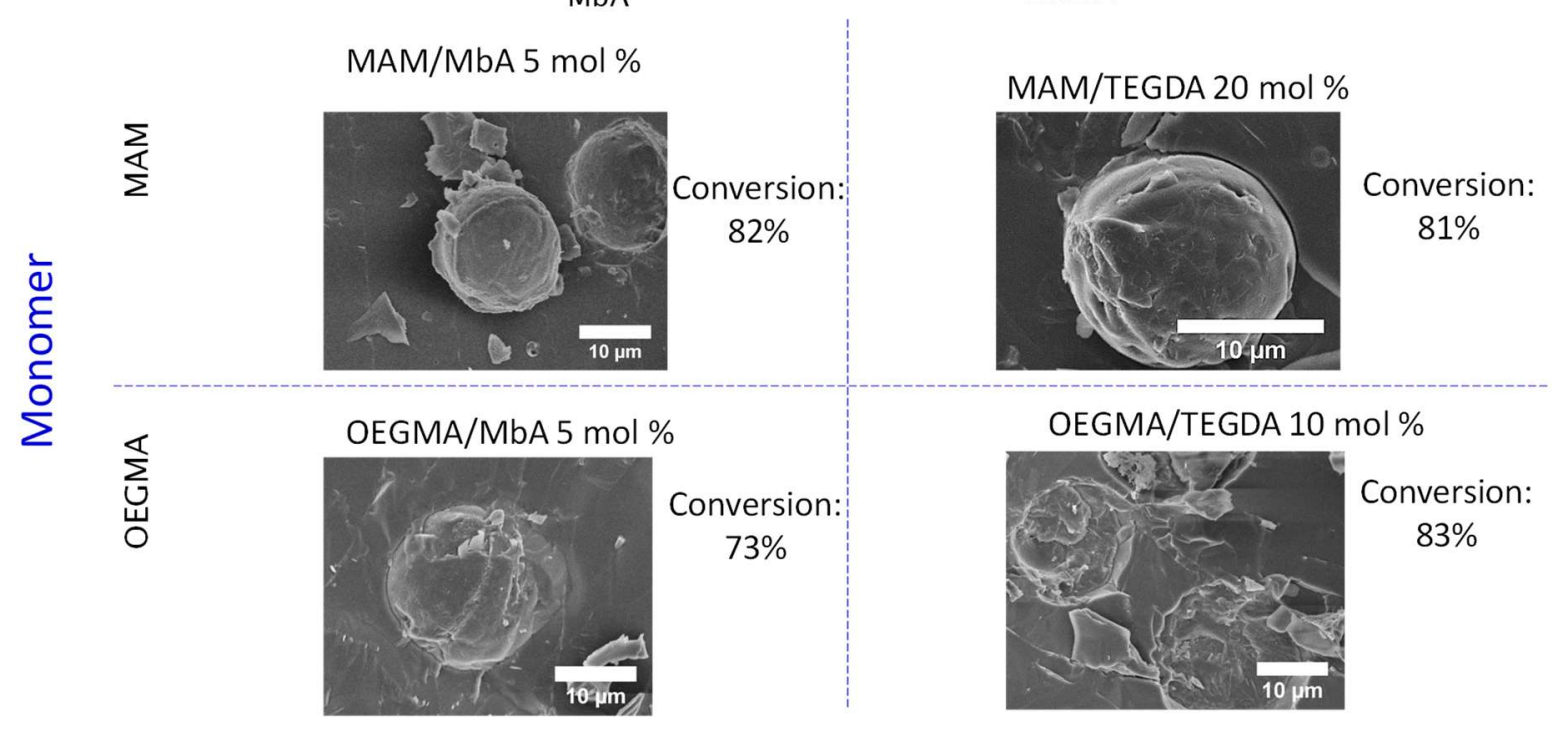

Figure 8: a) Addition of cross-linker in the double emulsion and b) obtained capsules. Adapted from [23]

4.3 Characterization of the capsules 
Once obtained it becomes interesting to characterize the capsules in terms of fragrance encapsulation efficiency and release in various conditions.

\section{Total amount of fragrance after polymerization and encapsulation efficiency}

The capsules are diluted with ethanol that is thought to remove all the encapsulated and non-encapsulated fragrance. After 30 minutes of contact, ethanol containing fragrance is removed and analyzed by gas chromatography. This protocol is the same as the one classically used for core-shell capsules. Indeed ethanol and fragrance are miscible and ethanol is a good solvent for the polymer shell that swells allowing fragrance recovery. The proportion of recovered fragrance in ethanol with respect to the initial amount of fragrance incorporated in the double emulsion is reported in Table 2.

Table 2: a) proportion of fragrance recovered in ethanol after polymerization and b) encapsulation efficiency as defined by (1).

\begin{tabular}{|c|c|c|c|}
\hline \multirow{2}{*}{\multicolumn{2}{|c|}{ a) }} & \multicolumn{2}{|c|}{ Cross-linker } \\
\hline & & $\mathrm{MbA}$ & TEGDA \\
\hline \multirow{2}{*}{ monomer } & MAM & $97.5 \%$ & $96.3 \%$ \\
\hline & OEGMA & $96.5 \%$ & $99.7 \%$ \\
\hline
\end{tabular}

\begin{tabular}{l|l|c|c|}
\cline { 3 - 4 } \multicolumn{2}{c|}{} & \multicolumn{2}{c|}{ Cross-linker } \\
\cline { 3 - 4 } \multicolumn{2}{c|}{} & MbA & TEGDA \\
\hline \multirow{2}{*}{ monomer } & MAM & $84.7 \%$ & $70.8 \%$ \\
\cline { 2 - 4 } & OEGMA & $80.6 \%$ & $80.9 \%$ \\
\hline
\end{tabular}

Among the fragrance extracted by ethanol, the proportion that is encapsulated can be determined using Equation 1, for that the non encapsulated fragrance is extracted using a dilution in IPM and a soft centrifugation. The results are reported in Table $2 \mathrm{~b}$. High encapsulation rates are achieved. 
Despite the high rates of recovery, a small fraction of fragrance is not present in ethanol. This may due to an incomplete recovery by ethanol linked to the matrix nature of the capsules making the polymer swelling more difficult. An alternate explanation could be evaporation during heating required to induce polymerization, interactions between monomers and fragrance molecules or degradation of some fragrance molecules during polymerization. To get better insight about the possible phenomenon, the recovered fragrance is analyzed by gas chromatography (figure 9 top) as well a pure fragrance heated 24 hours at $80^{\circ} \mathrm{C}$ and fragrance in contact with water containing KPS (demixed phases) also heated 24 hours at $80^{\circ} \mathrm{C}$ (Figure 9 bottom).

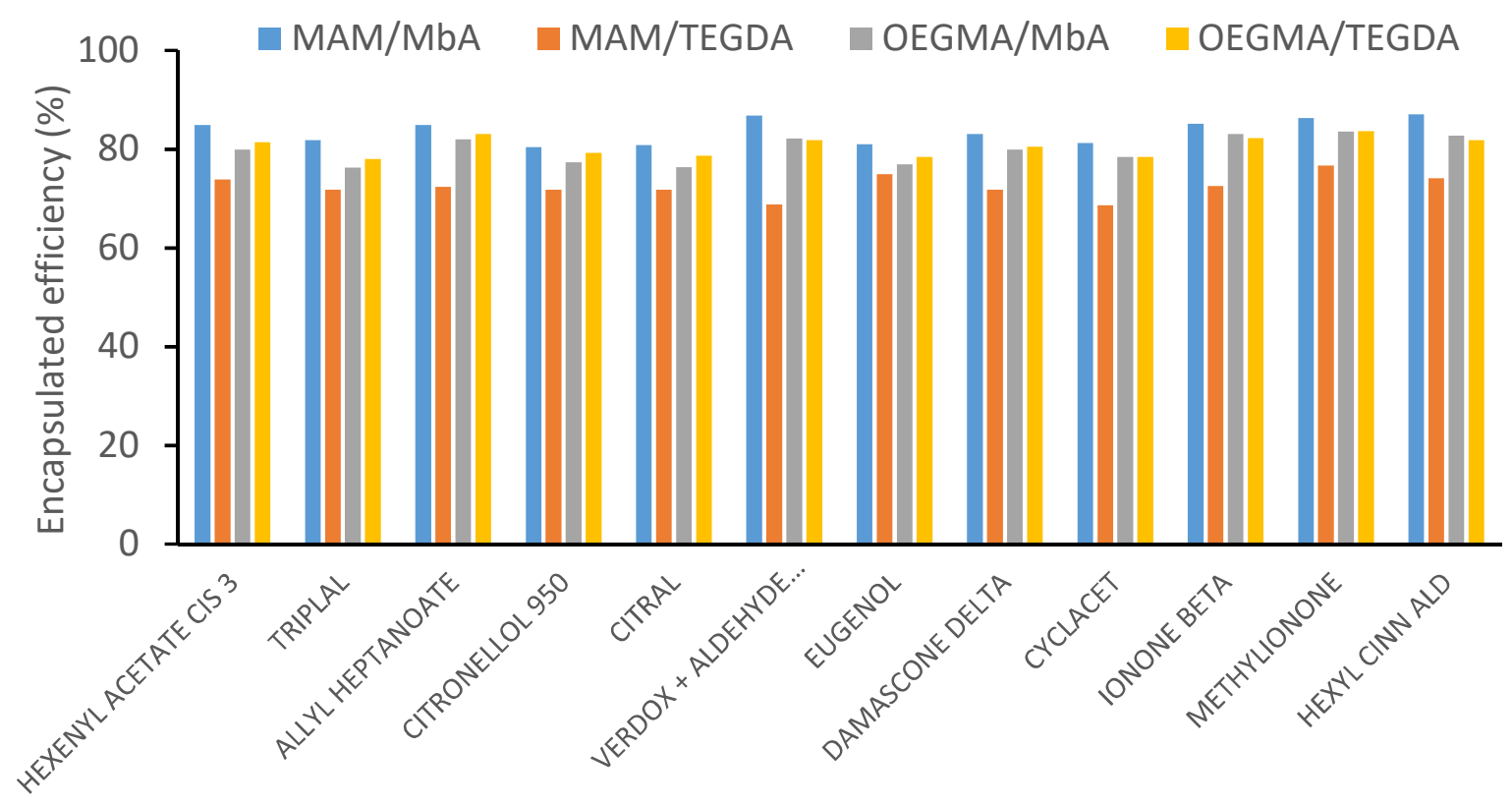




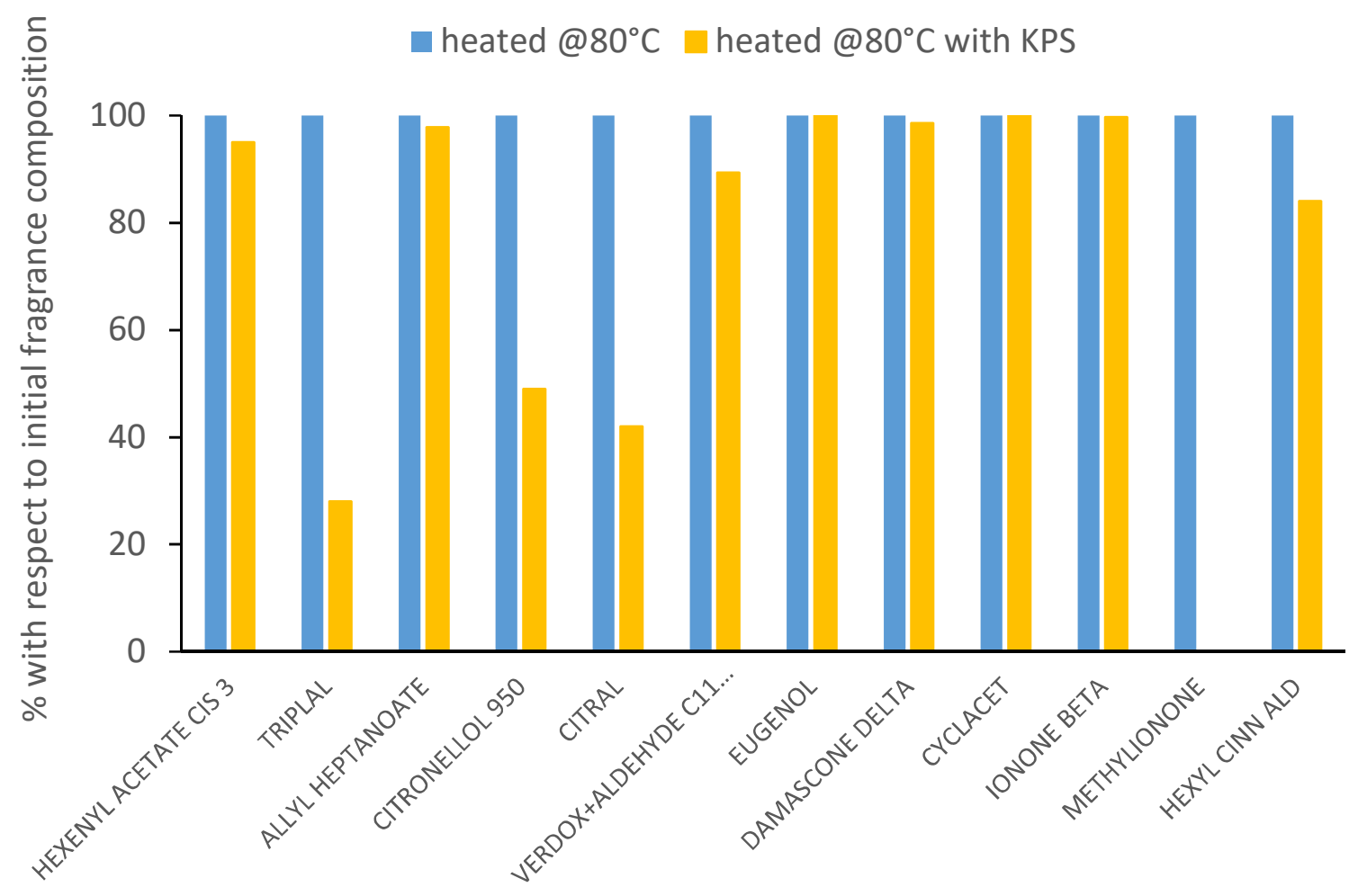

Figure 9: Fragrance analysis contained in the capsules (top) and control sample without capsules. Adapted from [23].

From Figure 9 (bottom), it can be seen that some molecules are very sensitive to the presence of KPS when heated: Triplal, Citronellol, Citral, Aldehyde C11 undecylenic and hexyl cinnamic acid in a much smaller extend. Nevertheless, these molecules are present in the capsules in a larger proportion (Figure 9 top) showing that the proposed strategy has a protective effect on them.

From the previous results, the fragrance composition after polymerization can also be plotted and compared to the initial one (Figure 10) 


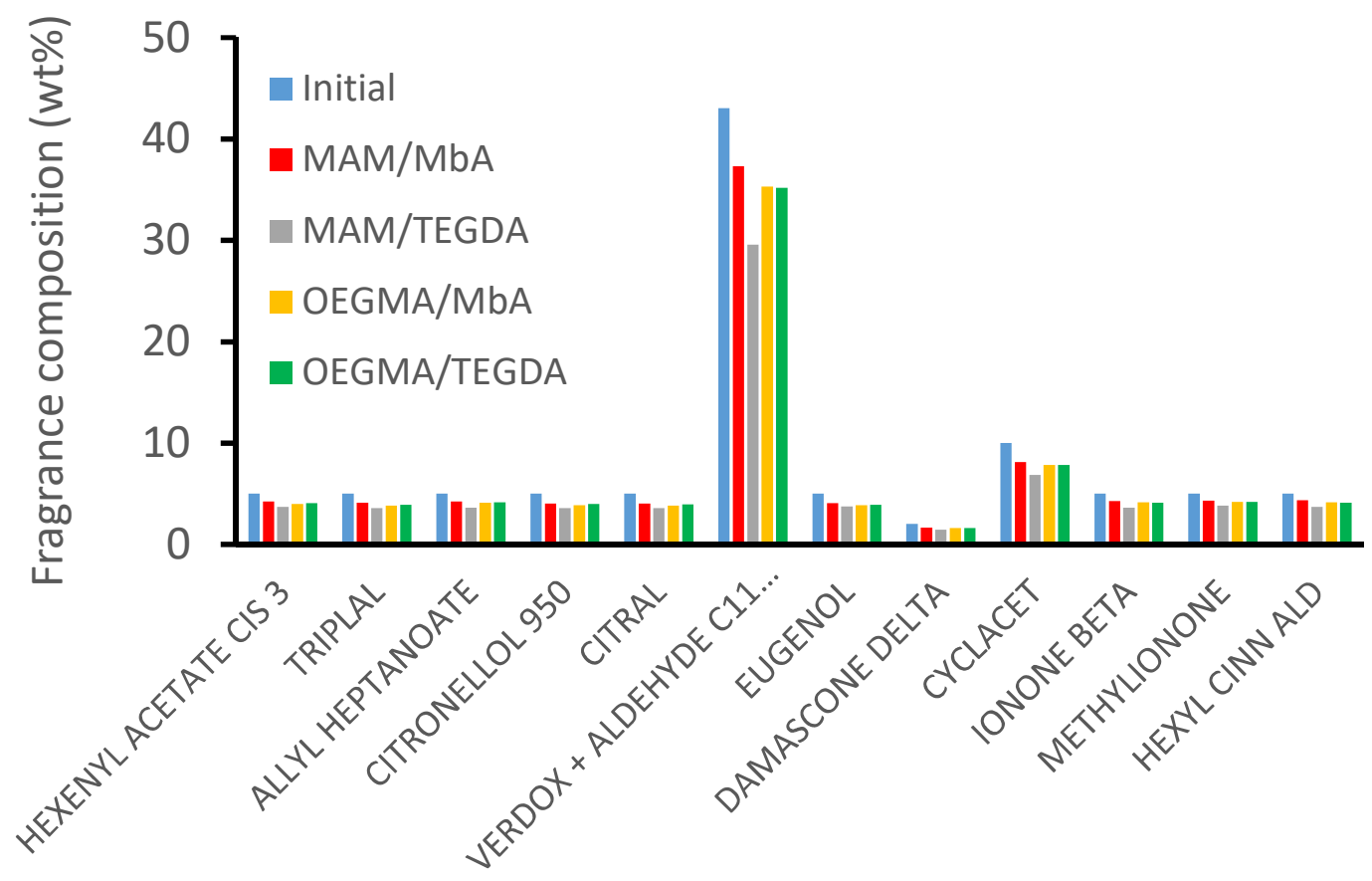

Figure 10: Composition of the fragrance in the capsules, derived from Figure 9 top and from the initial fragrance composition.

\section{Evolution of encapsulated fragrance with time in closed vials}

A small amount $(1 \mathrm{~g})$ of capsules is deposited on the bottom of $20 \mathrm{ml}$ vials with a lid. This means that a large volume of air occupies the vials. At regular time intervals, a small amount of the capsules is taken and diluted in IPM in order to measure the amount of fragrance still encapsulated. The results are normalized by the initial amount of fragrance (in the double emulsion formulation before polymerization). Note that possible escaped fragrance in the air of the vials is not considered so that the measure is not biased. The results are reported on Figure 11. 


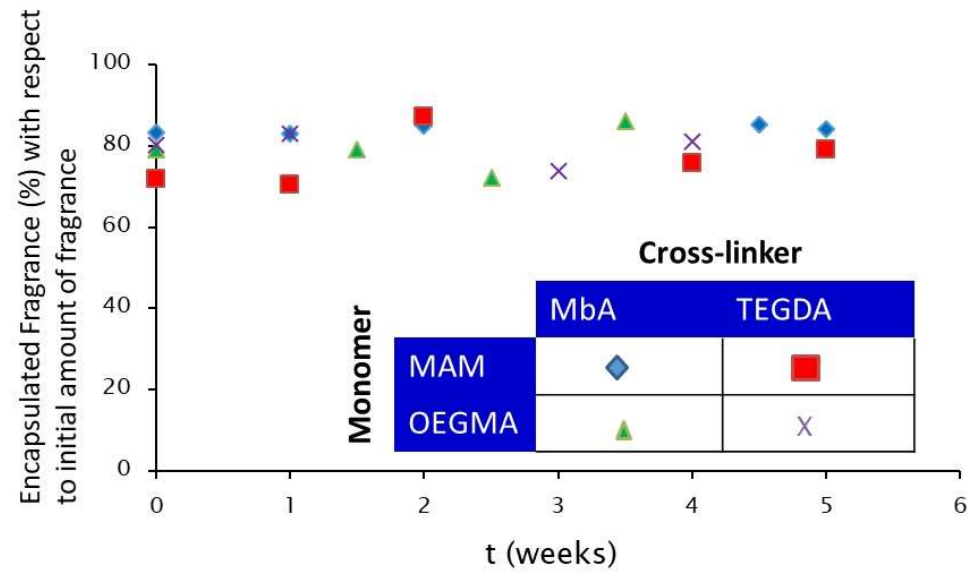

Figure 11: Evolution with time of the fragrance encapsulation rate (with respect to initial amount of fragrance). Adapted from [23].

No visible evolution over 5 weeks could be detected showing that fragrance is not released in close vials and that the capsules exhibit a high kinetic stability for storage.

In opposition for use, these capsules are required to release the encapsulated fragrance.

Evolution of encapsulated fragrance with time in open vials at 20 and $40^{\circ} \mathrm{C}$

The mass loss of capsules deposited on small cups without lid in a thermally controlled oven is measured as well as each of the constituting phase. The results are reported on Figure 12
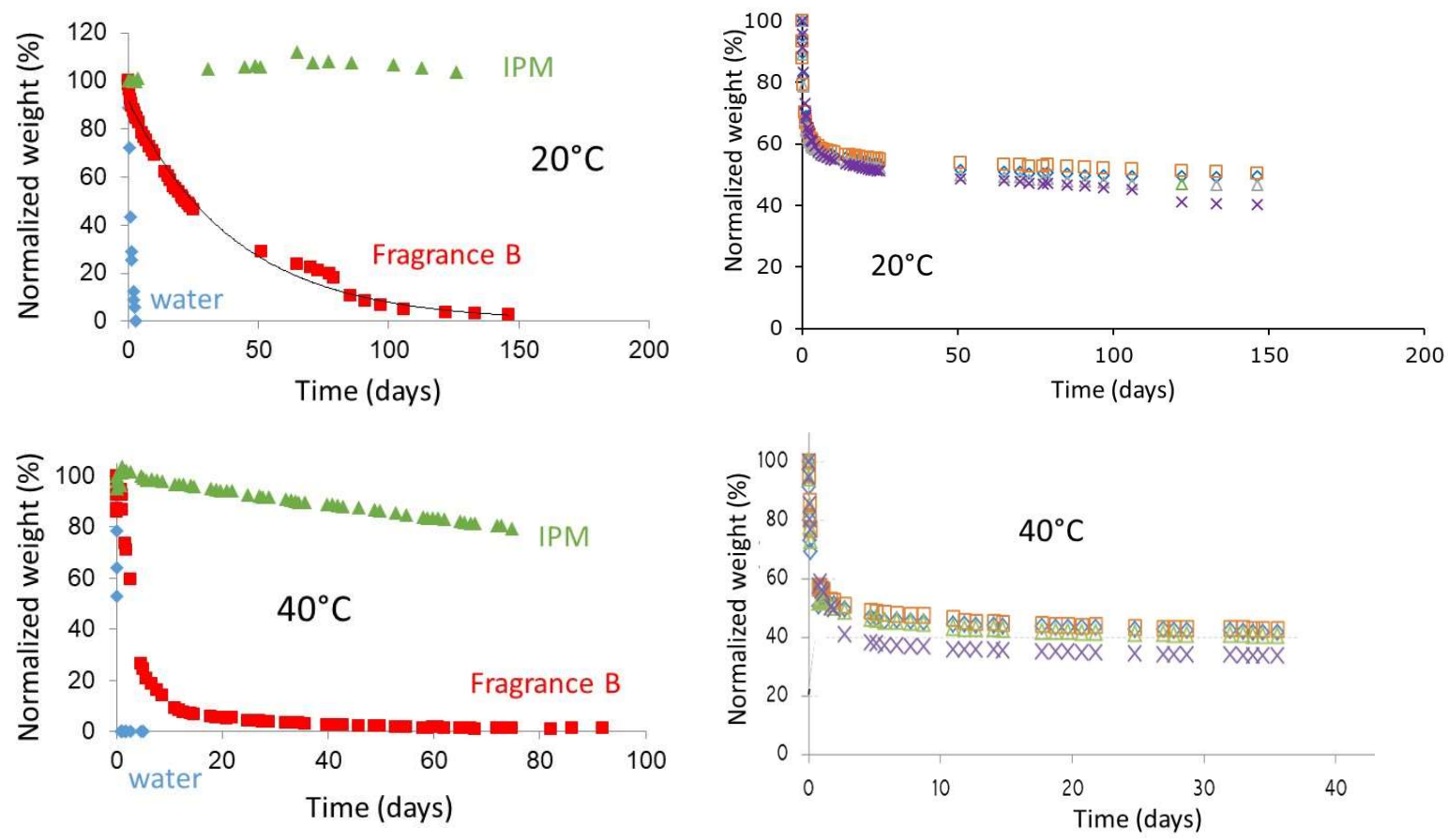
Figure 12: Evolution of the normalized weight at $20^{\circ} \mathrm{C}$ (top) and $40^{\circ} \mathrm{C}$ (bottom) for the isolated phases (left) and the capsules (right): $\diamond$ MAM/MbA, $\square$ MAM/TEGDA, $\triangle$ OEGMA/MbA and $x$ OEGMA/TEGDA Adapted from [23].

At $20^{\circ} \mathrm{C}$, a first fast decrease of the capsule weight is observed that can be attributed to the water loss, the second slowly loss is due to fragrance and it can be noted that the mass of IPM does not evolve at all over 150 days. At $40^{\circ} \mathrm{C}$, the same accelerated tendency is observed but IPM also evaporates. Taking into account the composition of the capsules and the water and when required the IPM losses, the fragrance release can be estimated for the various capsules. The results are reported as examples after 150 days at $20^{\circ} \mathrm{C}$ and after 40 days at $40^{\circ} \mathrm{C}$ on Figure 13.

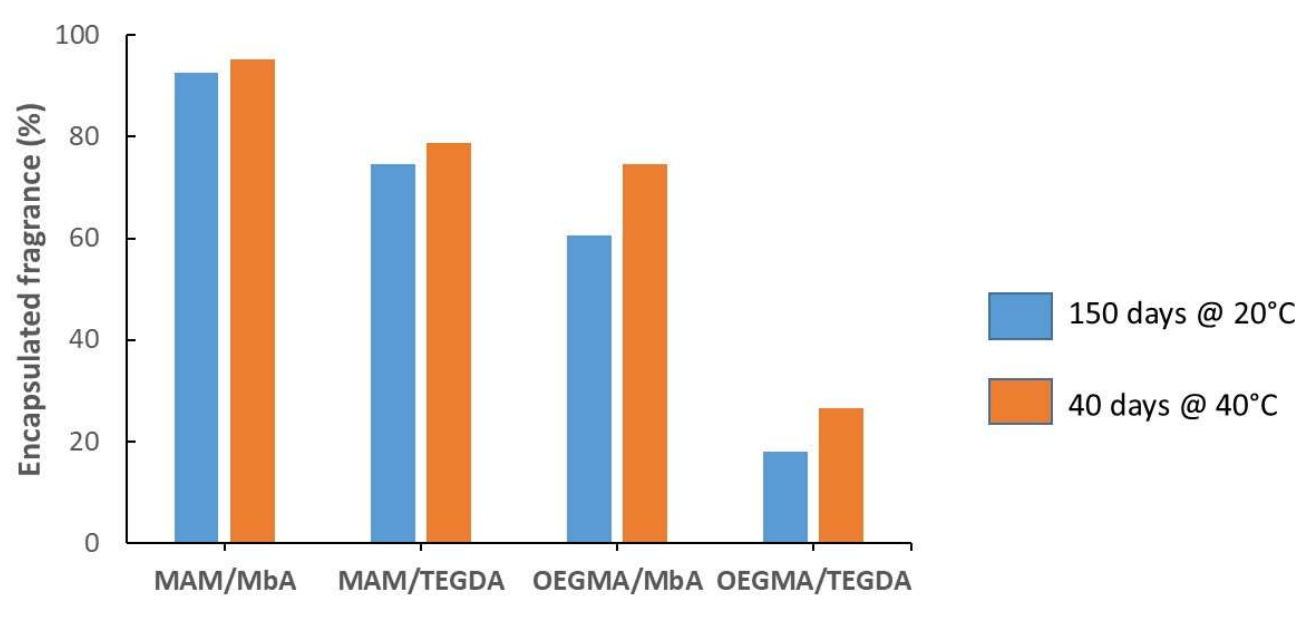

Figure 13: Proportion of the initial fragrance still encapsulated after 150 days at $20^{\circ} \mathrm{C}$ and after 40 days at $40^{\circ} \mathrm{C}$.

These experiments show that the proposed strategy allows tuning the release/retention properties of the capsules by an adequate choice of the monomers and cross-linkers. In figure 13, the capsules are sorted out from left to right from the highest fragrance retention (lowest fragrance release) to the lowest fragrance retention (highest fragrance release).

\section{Mechanical resistance}

It is worth noticing that all capsules resisted to a $10^{-3} \mathrm{~Pa}$ vacuum during 12 hours without any rupture or morphology evolution. Then a home-made set-up [23] allowed simultaneously applying a compression (using a load of $100 \mathrm{~g}$ corresponding to $780 \mathrm{~Pa}$ and $150 \mathrm{~g}$ corresponding to $1170 \mathrm{~Pa}$ ) and taking a part of the recovered fragrance in an excess of IPM. Fragrance is release by compression as plotted in Figure 14 


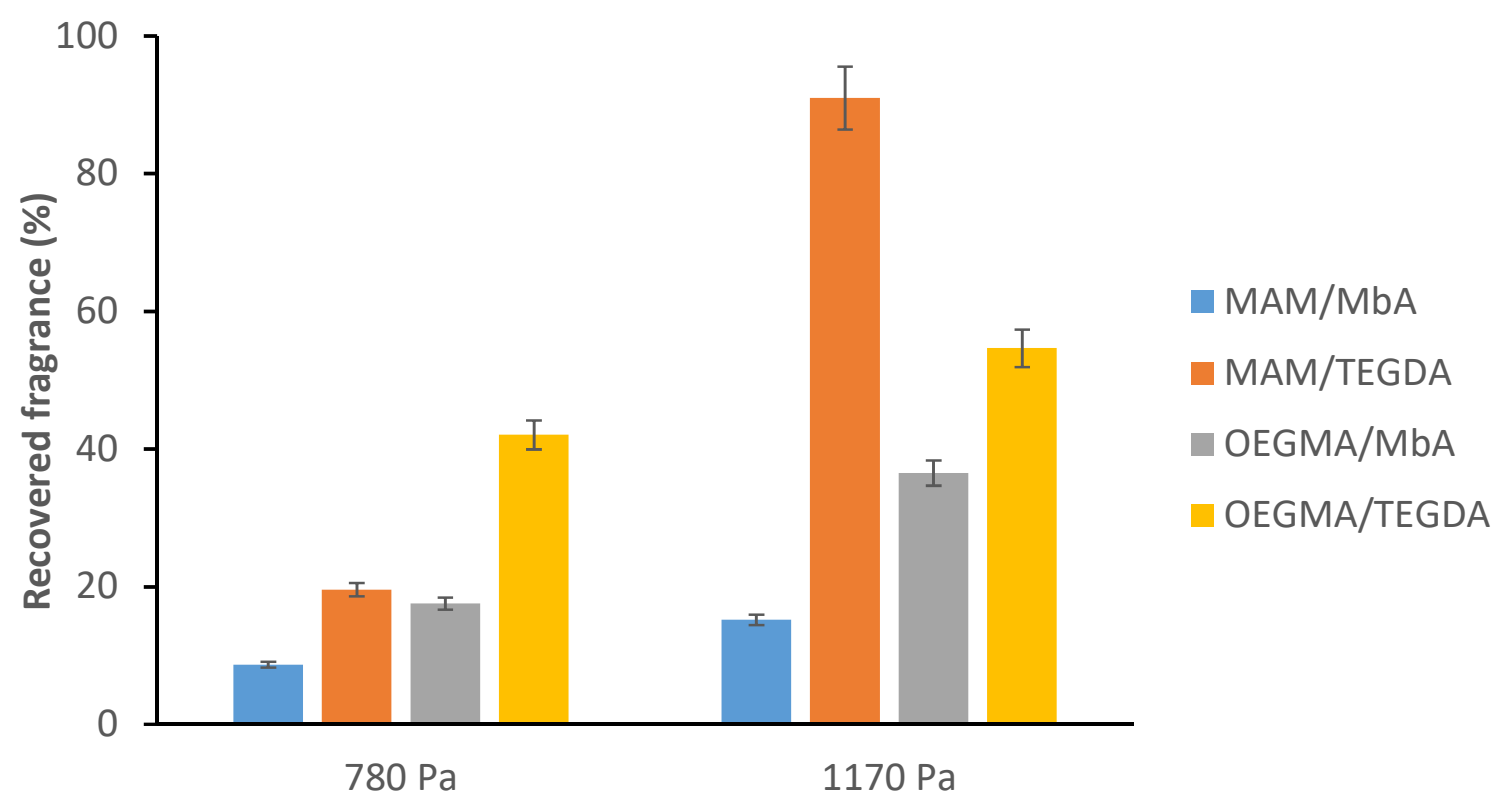

Figure 14: Amount of released fragrance under compression normalized by the initial amount of fragrance. Adapted from [23].

When the load is removed and the capsules can relax, no fracture and no morphology evolution can be observed, showing the elastic nature of the capsules that behave as small sponges.

\section{Conclusion}

Herein we presented a novel strategy to elaborate polymer capsules containing multicomponent fragrance from the formulation of double fragrance-in-aqueous phase-in-perfumery solvent (isopropyl myristate) emulsions. This strategy aims at compartmentalizing the fragrance and the polymerization locus. The selected double emulsion formulation resists changes in fragrance composition in the innermost droplets and addition of monomers, cross-linkers and initiator in the aqueous intermediate phase. This allows polymerizing and cross-linking this intermediate phase to elaboration a tri-dimensional network achieving matrix capsules. This strategy is also versatile as various monomers, cross-linkers may be used, and the obtained properties are tunable. Indeed, a judicious choice of monomers and cross-linkers allows adjusting the fragrance passive release. In addition to the fragrance diffusion across the capsules, the release may also be triggered by applying a compression leading to an additional boost effect. All the proposed capsules exhibit morphologies that are resistant to compression, no fracture has been observed and the polymer capsules act as small sponges. Moreover, unexpectedly, beginning with a double emulsion dispersed in a perfumery oil, no transfer of the 
capsules into water was necessary after polymerization as the capsules were already dispersible in water owing to the affinity of the polymer towards water.

\section{Acknowledgment}

The authors would like to thank Olivier Anthony for fruitfull discussions, Eric Laurichesse for his technical support, Maud Bassereau for her support in interpreting some GC chromatograms and Takasago Europe Perfumery Laboratory for assembling the fragrances. The ANRT, Takasago Europe Perfumery Laboratory, CNRS, Université de Bordeaux and INP are acknowledged for financial support (contract number 2014/1309).

\section{Conflict of Interest}

The authors declare no conflict of interest that could influence or impart bias on the work

\section{References}

1. Doane, W.M., Encapsulation of pesticides in starch by extrusion. Industrial Crops and Products, 1992. 1(2-4): p. 83-87 DOI: 10.1016/0926-6690(92)90004-F.

2. Tsuiji, K., Microencapsulation of pesticides and their improved handling safety. Journal of Microencapsulation, 2001. 18(2): p. 137-147 DOI: 10.1080/026520401750063856.

3. Zuidam, N.J. and V.A. Nedović, Encapsulation technologies for active food ingredients and food processing. Encapsulation Technologies for Active Food Ingredients and Food Processing. 2010. 1-400.

4. Nelson, G., Application of microencapsulation in textiles. International Journal of Pharmaceutics, 2002. 242(1-2): p. 55-62 DOI: 10.1016/S0378-5173(02)00141-2.

5. Singh, M.N., K.S.Y. Hemant, M. Ram, and H.G. Shivakumar, Microencapsulation: A promising technique for controlled drug delivery. Research in Pharmaceutical Sciences, 2010. 5(2): p. 65-77.

6. Konuklu, Y., M. Ostry, H.O. Paksoy, and P. Charvat, Review on using microencapsulated phase change materials (PCM) in building applications. Energy and Buildings, 2015. 106: p. 134-155 DOI: 10.1016/j.enbuild.2015.07.019.

7. Warr, J., T. Ribaut, E. Aussant, O. Antony, and S. Fraser, New microcapsules 2013, Patent EP20130305075 (A2).

8. Warr, J., T. Ribaut, O. Anthony, and S. Fraser, Microcapsules, 2015, Patent EP2832442 (A1).

9. Warr, J., T. Ribaut, O. Anthony, and S. Fraser, Microcapsules, 2015, Patent EP2832441 (A1).

10. Warr, J., T. Ribaut, O. Anthony, and S. Fraser, Microcapsules, 2015, Patent EP2832440 (A1).

11. Jahns, E., D. Boeckh, W. Bertleff, and P. Neumann, Microcapsule preparations and detergents and cleaning agents containing microcapsules, 2005, Patent Brevet US 6951836 B2.

12. Jahns, E. and B. Dames, Microcapsules, the production and use thereof, 1997, Patent Brevet US 5596051. 
13. Jacquemond, M., N. Jeckelmann, L. Ouali, and O.P. Haefliger, Perfume-containing polyurea microcapsules with undetectable levels of free isocyanates. Journal of Applied Polymer Science, 2009. 114(5): p. 3074-3080 DOI: 10.1002/app.30857.

14. Kuo, S.-W., H.-C. Kao, and F.-C. Chang, Thermal behavior and specific interaction in high glass transition temperature PMMA copolymer. Polymer, 2003. 44(22): p. 68736882 DOI: 10.1016/j.polymer.2003.08.026.

15. Bancroft, W.D., The theory of emulsification, $V$. The Journal of Physical Chemistry, 1913. 17(6): p. 501-519.

16. Mabille, C., F. Leal-Calderon, J. Bibette, and V. Schmitt, Monodisperse fragmentation in emulsions: Mechanisms and kinetics. Europhysics Letters (EPL), 2003. 61(5): p. 708714 DOI: 10.1209/epl/i2003-00133-6.

17. Mabille, C., V. Schmitt, P. Gorria, F. Leal Calderon, V. Faye, B. Deminière, and J. Bibette, Rheological and Shearing Conditions for the Preparation of Monodisperse Emulsions. Langmuir, 2000. 16(2): p. 422-429 DOI: 10.1021/la990850w.

18. Stasse, M., E. Laurichesse, T. Ribaut, O. Anthony, V. Héroguez, and V. Schmitt, Formulation of concentrated oil-in-water-in-oil double emulsions for fragrance encapsulation. Colloids and Surfaces A: Physicochemical and Engineering Aspects, 2020. 592: p. 124564.

19. Nollet, M., E. Laurichesse, S. Besse, O. Soubabère, and V. Schmitt, Determination of Formulation Conditions Allowing Double Emulsions Stabilized by PGPR and Sodium Caseinate to Be Used as Capsules. Langmuir, 2018. 34(8): p. 2823-2833 DOI: 10.1021/acs.langmuir.7b04085.

20. Taylor, P., Ostwald ripening in emulsions. Advances in colloid and interface science, 1998. 75(2): p. 107-163.

21. Rodrigues, S.N., I.M. Martins, I.P. Fernandes, P.B. Gomes, V.G. Mata, M.F. Barreiro, and A.E. Rodrigues, Scentfashion ${ }^{\circledR}$ : Microencapsulated perfumes for textile application. Chemical Engineering Journal, 2009. 149(1): p. 463-472 DOI: https://doi.org/10.1016/j.cej.2009.02.021.

22. Stasse, M., T. Ribaut, V. Schmitt, and V. Héroguez, Encapsulation of lipophilic fragrance by polymerization of the intermediate aqueous phase of an oil-in-water-inoil $(\mathrm{O} / \mathrm{W} / \mathrm{O})$ double emulsion. Polymer Chemistry, 2019. 10: p. 4154-4162 DOI: 10.1039/C9PY00528E.

23. Stasse, M., E. Laurichesse, M. Vandroux, T. Ribaut, V. Héroguez, and V. Schmitt, Cross-linking of double oil-in-water-in-oil emulsions: a powerful way to obtain microcapsules for with attractive mechanical properties, application to fragrance encapsulation. submitted. 